\title{
Recent innovation of geospatial information technology to support disaster risk management and responses
}

\author{
Hiroshi $\mathrm{UNE}^{\mathrm{a}}$ and Takayuki NAKANO ${ }^{\mathrm{a}}$ \\ ${ }^{a}$ Geospatial Information Authority of Japan; une-h96fm@mlit.go.jp, nakano-t96fj@mlit.go.jp
}

\begin{abstract}
Geographic location is one of the most fundamental and indispensable information elements in the field of disaster response and prevention. For example, in the case of the Tohoku Earthquake in 2011, aerial photos taken immediately after the earthquake greatly improved information sharing among different government offices and facilitated rescue and recovery operations, and maps prepared after the disaster assisted in the rapid reconstruction of affected local communities. Thanks to the recent development of geospatial information technology, this information has become more essential for disaster response activities. Advancements in web mapping technology allows us to better understand the situation by overlaying various location-specific data on base maps on the web and specifying the areas on which activities should be focused. Through 3-D modelling technology, we can have a more realis-tic understanding of the relationship between disaster and topography. Geospatial information technology can sup-port proper preparation and emergency responses against disasters by individuals and local communities through hazard mapping and other information services using mobile devices. Thus, geospatial information technology is playing a more vital role on all stages of disaster risk management and responses. In acknowledging geospatial in-formation's vital role in disaster risk reduction, the Sendai Framework for Disaster Risk Reduction 2015-2030, adopted at the Third United Nations World Conference on Disaster Risk Reduction, repeatedly reveals the importance of utilizing geospatial information technology for disaster risk reduction. This presentation aims to report the recent practical applications of geospatial information technology for disaster risk management and responses.
\end{abstract}

Keywords: Disaster Reduction, Tohoku Earthquake, Geospatial Information, Sendai Framework

\section{Role of geospatial information for disaster risk reduction as exemplified during the Tohoku Earthquake in 2011}

Geographic location is one of the most fundamental and indispensable information elements in the field of disaster response and prevention. Without geospatial information, the effect of measures or actions for disaster risk reduction would be quite limited. The recent development of geospatial information technology has enabled decision makers and the general public to employ geospatial information and to integrate it with other information in an easy-to-use and affordable manner in every phase of the disaster management cycle, including disaster risk assessment, emergency response operations during disasters, and "build back better" recovery from disaster damage at any time and place (Fig. 1).

The Geospatial Information Authority of Japan (hereinafter "GSI") is the only national governmental organization in Japan for the production and coordination of basic geospatial information. Japanese Basic Act for Disaster Countermeasures designates relevant government agencies and other organizations for disaster response operations including GSI having legal authority to address disasters. In 2012, based on the lessons learned regarding the usefulness of geospatial information in the disaster responses to the 2011 off the Pacific coast of Tohoku Earthquake (hereinafter "the Tohoku Earthquake"), the above act was amended to include a provision for maximizing the use of geospatial information in the collection and communication of information concerning disaster prevention, reflecting greater expectations from the government for the geospatial community to promptly provide proper geospatial information.

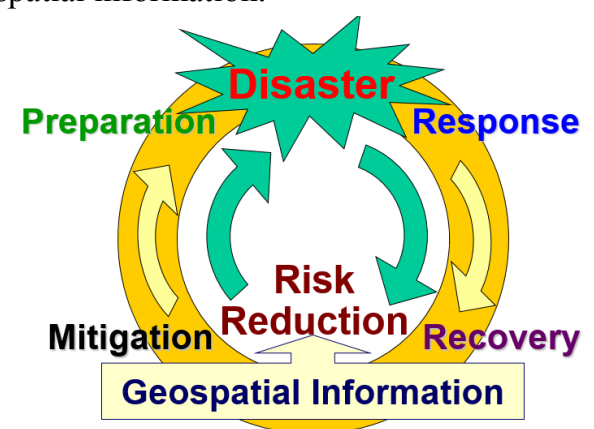

Figure 1. Disaster management cycle 


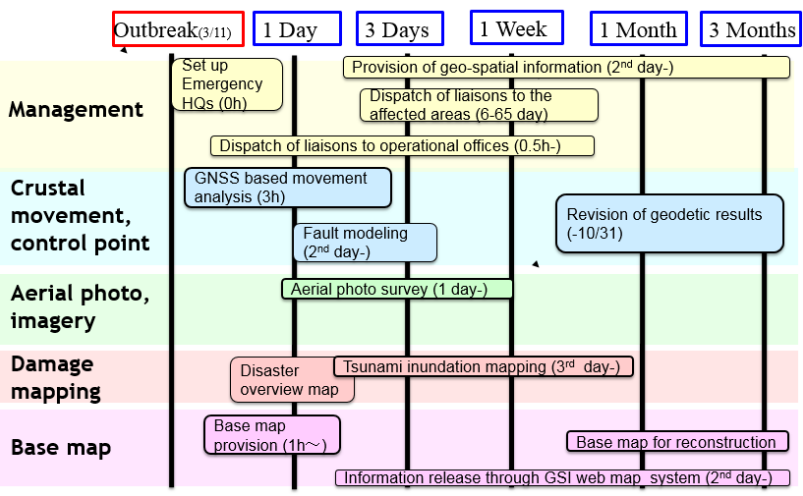

Figure 2. Emergency response activities of GSI

The Tohoku Earthquake took place at 2:46 PM local time on March 11, 2011. The epicenter was located about 130 $\mathrm{km}$ east off the Tohoku coast. Its magnitude, 9.0, was the largest observed in Japanese history and was the fourth largest in the world since 1900. In addition to terrible shaking, the main shock brought a gigantic tsunami wave over 10 meters high with a maximum run-up height of 40 meters over a wide range of the Pacific coast in the Tohoku region.

Just after the occurrence of the Tohoku Earthquake, the GSI went into emergency mode and immediately set up an emergency headquarters (EHQ) to serve as the decision-making body for disaster operations. The first EHQ meeting was held at 3:10 PM, within thirty minutes of the main shock. In accordance with the decisions of the EHQ, the GSI took various actions to support the victims and damaged regions by surveying and providing geospatial information, such as distributing maps and aerial photos, observing crustal deformation, mapping inundation areas, and fully collaborating with private surveying companies and related organizations. Among its efforts, three major emergency response activities were 1) the provision of maps, 2) the detection of ground surface movement using a Global Navigation Satellite System (GNSS) control point network, and 3) conducting aerial surveys and interpreting photos of damaged areas (Fig. 2).

Immediately after the Tohoku Earthquake, the GSI delivered base maps of the damaged areas to relevant government offices, starting with small-scale maps sent to central government offices via the Internet or in the form of paper maps. In addition to the map data, the GSI dispatched liaison staff to those offices to provide services for preparing maps of synoptic views of the damage and recovery status for their briefings. The GSI also dispatched staff to onsite headquarters of the government for disaster management with map data and equipment, including computers and large-sized printers to provide paper maps to meet the needs of officials in charge of rescue and relief operations.

As for the detection of ground surface movement, the GSI made the best use of its GNSS control point network. Since 1995, the GSI has utilized the GNSS Earth Observation Network System (GEONET), with more than 1,200 GNSS continuous observation stations throughout the country for location reference services and detection of crustal deformation. After the main shock of the Tohoku Earthquake, the GSI used its best efforts to obtain initial results of ground surface movements within a few hours. Ultimately, an analysis of the observed data revealed that the maximum movement was 5.3 meters in the horizontal direction and minus 1.2 meters vertically (Fig. 3).
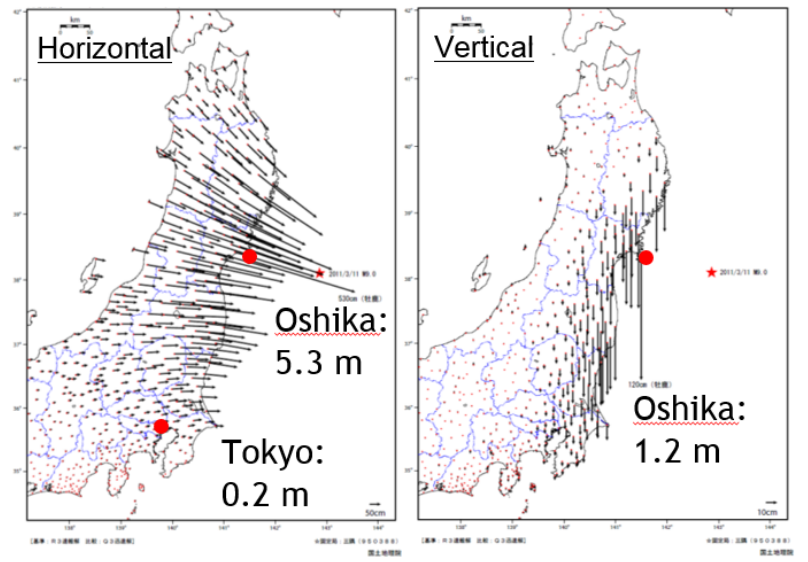

Figure 3. Ground surface movements observed by GEONET (left side: horizontal deformation; right side: vertical deformation)

In order to get a grasp of the situation with regard to damage, an aerial survey was conducted starting the next day, covering the whole coastal region, except the area around the crippled nuclear reactors. The GSI possesses its own aircraft, the "Kunikaze 3," and a special crew team for aerial surveys. However, the damaged area that needed to be covered was assumed to be extremely large, obviously far beyond the coverage ability of a single aircraft. In the event the GSI is not available to implement emergency tasks when a disaster occurs, in 2005, it entered into an "Agreement on Emergency Photography for Disaster Occurrence" with private surveying companies to receive assistance in efficiently conducting emergency photography. Immediately after the earthquake, the GSI decided to request collaboration based on this agreement to conduct coordinated emergency surveys. The weather during the next few days was very cooperative, and the air crew teams were able to take aerial photos of significantly dam-aged areas on March 12 and 13. The remaining areas were covered in subsequent survey missions by the GSI. This collaborative framework will need to function well in the event of expected, future large-scale earthquakes and tsunamis such as the Nankai Trough Earthquake. In addition, the GSI is promoting the development of applications for the latest remote-sensing technology such as synthetic aperture radar imaging from aerial platforms in the event of disasters occurring during bad weather or at night. As a result of the emergency surveys, color aerial photographs of approximately $6,900 \mathrm{~km} 2$ of the affected areas were taken, mostly on a scale of 1:20,000 (Fig. 4). The acquired data were processed as quickly as possible, mobilizing resources through the night. Aerial photo data were delivered to major governmental 
organizations and uploaded to the GSI's website the next day, March 14. The results enabled comparisons with photos taken before the earthquake to highlight the damage caused by the tsunamis (Fig. 5).

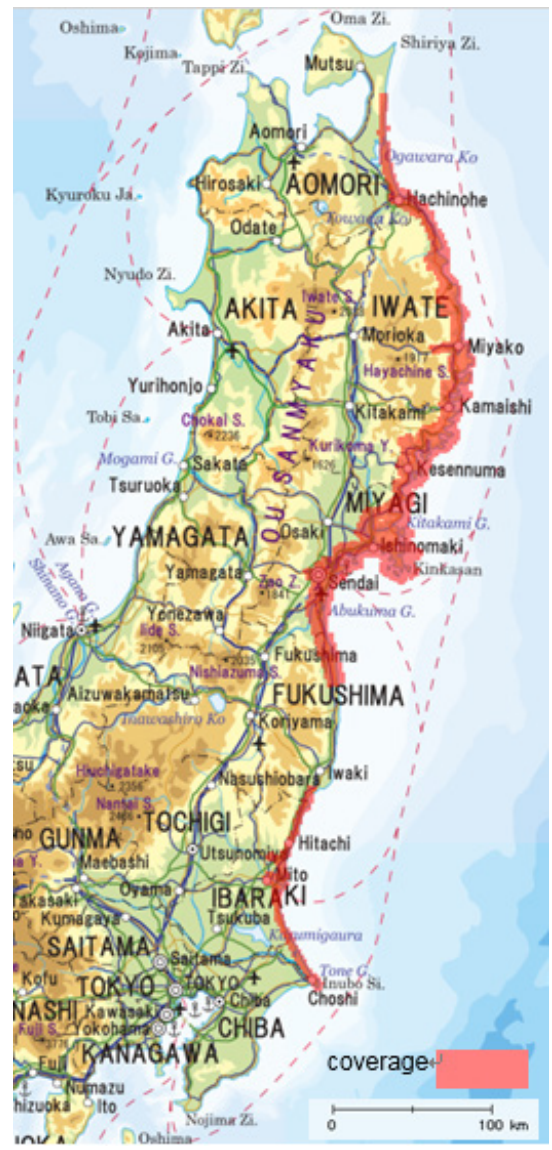

Figure 4 . Areas covered by the aerial photographs
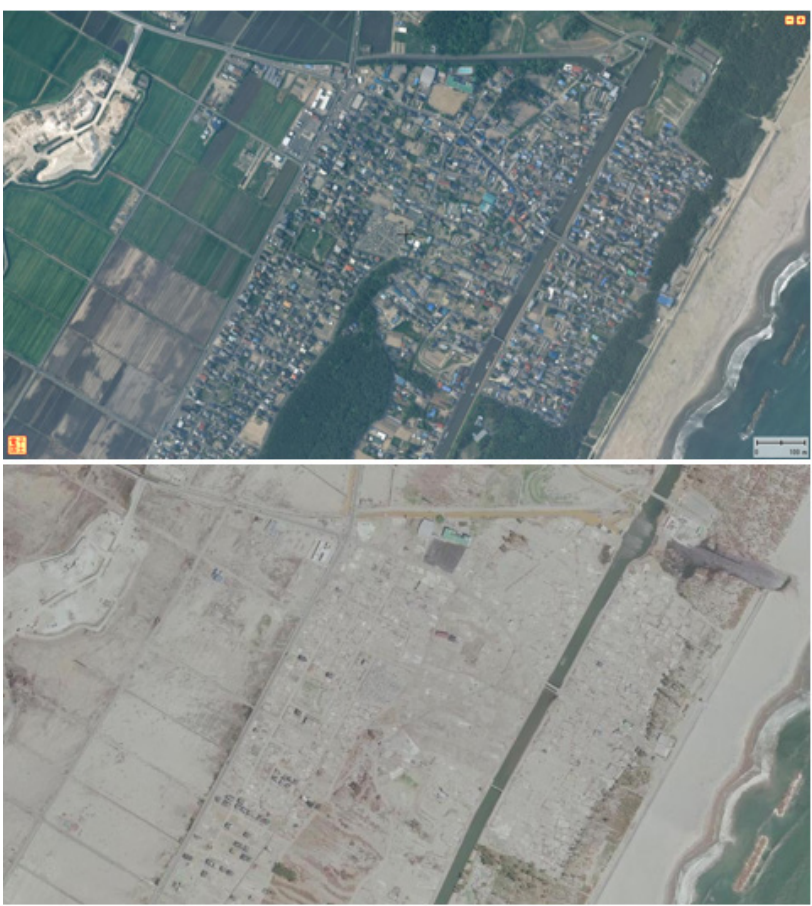

Figure 5. An example of aerial photo images of a damaged area near Natori City taken after the Tohoku Earthquake by the GSI. (upper photo: before the tsunami; lower photo: after the tsunami)

The GSI created a "tsunami flood area overview map" to ascertain the extent of the tsunami damage. As the aerial photos came in, the GSI staff started working on photo interpretations of the inundated areas and compiled the results on 1:25,000 topographic maps using GIS software. Then, the results were digitally copied to 1:100,000 scale maps for a better understanding of the whole picture of the damage. These were uploaded on the GSI's web-site (Fig. 6). The results showed that the inundated areas totaled as much as $561 \mathrm{~km} 2$.

A few weeks after the earthquake, the GSI commence two projects for recovery of the damaged areas. The first was a resurvey of the control points. Due to the large ground displacement, it was imperative to resurvey the control points to revise their coordinates so that reconstruction activities could be initiated. In addition, for reconstruction planning, the GSI prepared large-scale base maps on a scale of 1:2,500 for the damaged areas.

As for the resurvey of the control points, approximately 1,900 triangulation points and about the same number of bench marks were resurveyed. For approximately 43,000 control points that were not directly resurveyed, coordinate correction parameters were provided and revised coordinates were calculated. In addition, because the horizontal and vertical origins of the country's geodetic coordinate system located in Tokyo were displaced, their coordinates were also revised. This whole process was completed in less than eight months after the earthquake and contributed significantly to the recovery process.
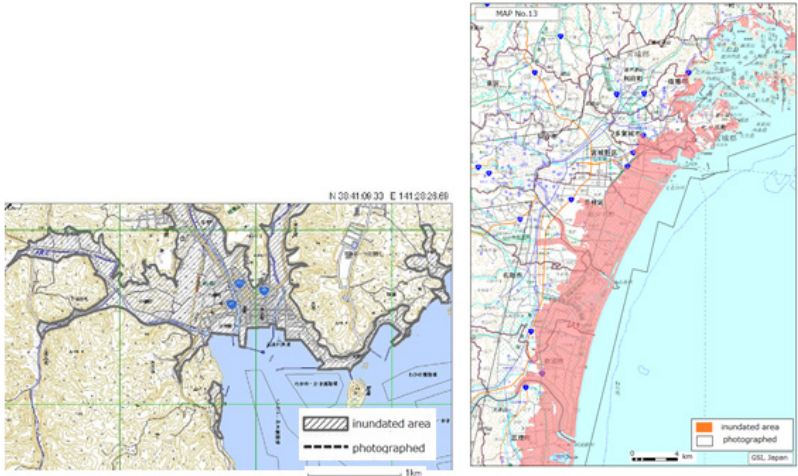

Figure 6. An example of a "tsunami flood area overview map" compiled on a 1:25,000 topographic map (left) and digitally copied to a 1:100,000 map (right)

\section{Recent innovation of geospatial information for disaster risk reduction}

Recent years have seen rapid and significant development and dissemination of innovative technologies that are relevant to geospatial applications, including satellite positioning, wireless Internet access, satellite-based and in situ Earth observation, web mapping, unmanned aerial vehicles (UAVs), and near real-time Big Data analysis. Thanks to recent developments in geospatial information technology, this information has become essential for disaster response activities. 
Advancements in web mapping technology allow us to better understand the situation by overlaying various location-specific data on base maps on the web and specifying the areas on which the activities should be focused. Figure 7 shows an example from the case of a debris flow disaster in Hiroshima City in 2014. Various georeferenced data related to rescue and disaster response activities, such as affected areas, refuges, and work sites, were overlaid on web-based digital maps provided by the GSI ("GSI Maps").

Distribution of reasonable image processing software applying Structure from Motion (SfM) and the MultiView Stereo (MVS) theory brought about new disaster information acquisition methods. This enables us to create seam-less orthographic images from oblique photos, which means that in order to take vertical aerial photographs to grasp a disaster situation, we no longer have to wait for weather recovery after flooding caused by heavy rain or the end of volcanic activity after an eruption (Fig. 8).

Additionally, 3-D modelling technology gives us a realistic understanding of the relationship between disaster and topography. Figure 9 present a 3-D image of a landslide triggered by the Kumamoto Earthquake in 2016. The 3-D presentation function is automatic on "GSI Maps."

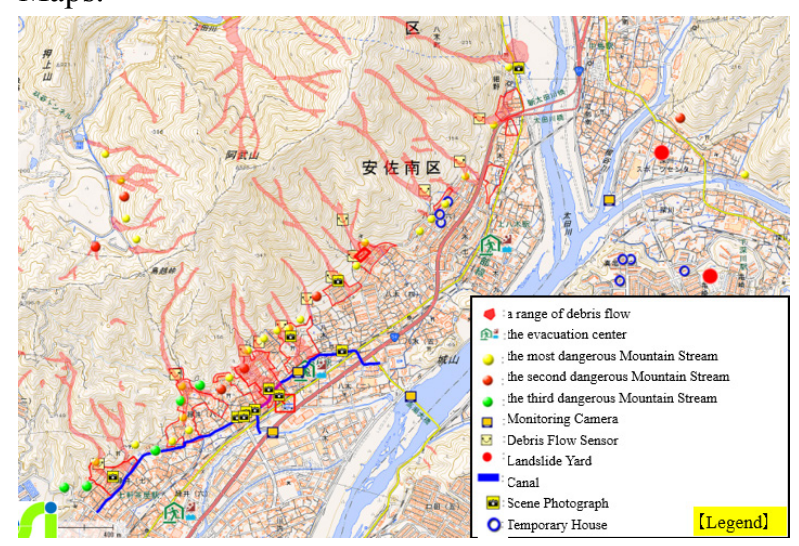

Figure 7. Integration of key disaster response information for a debris flow in Hiroshima in 2014

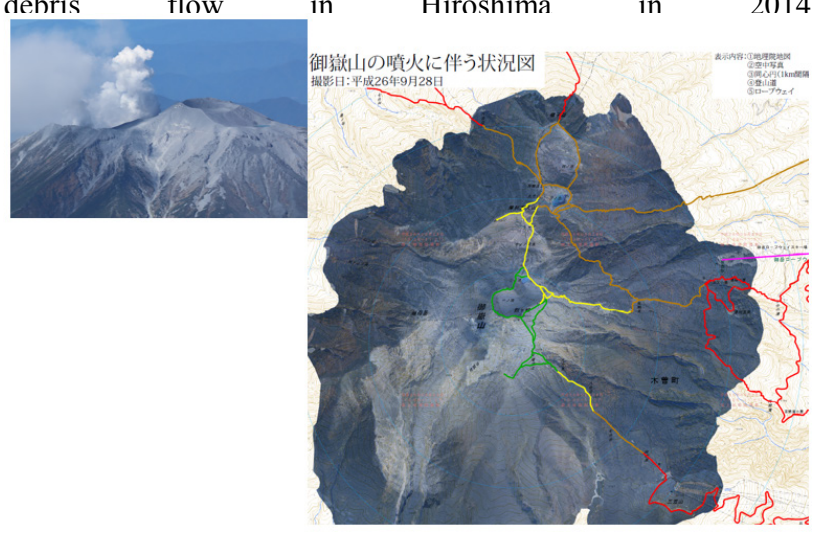

Figure 8. Orthographic image created from oblique photos from the Mt. Ontake eruption in 2014

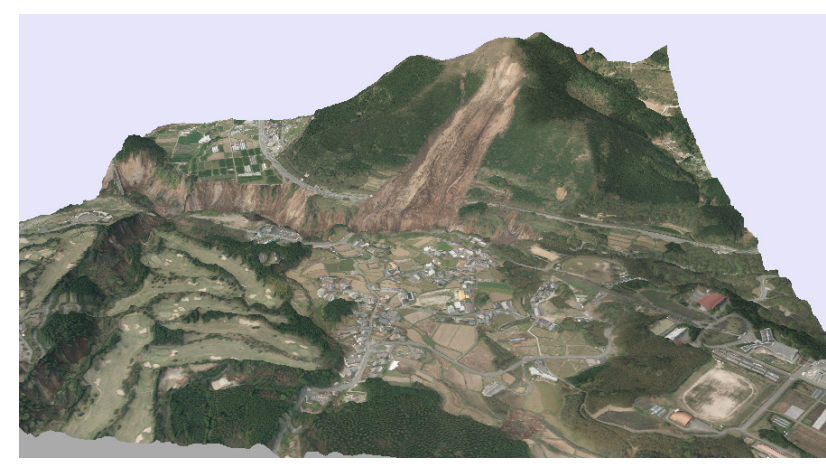

Figure 9. A 3-D image of a landslide triggered by the Kumamoto Earthquake in 2016

\section{Conclusions}

Geospatial information technology can support proper preparation and emergency responses against disasters by individuals and local communities through hazard mapping and other information services using mobile devices. For instance, mobile phone applications to assist in the evacuation of residents and a network analysis system of evacuation routes as a risk communication tool for local communities are becoming increasingly common.

Thus, geospatial information technology, the result of geography and cartography, is now playing a more vital role on all stages of disaster risk management and responses. In acknowledging the vital role of geospatial information for disaster risk reduction, the Sendai Framework for Disaster Risk Reduction 2015-2030, adopted at the Third United Nations World Conference on Disaster Risk Reduction, held in March 2015 in Sendai, Japan (Miyagi Prefecture), reveals the importance of utilizing geospatial information technology for disaster risk reduction.

\section{Acknowledgements}

The author would like to thank Editage (www.editage.jp) for English language editing. 